\title{
Medicamentos versátiles: buscando balas mágicas
}

\author{
Versatile drugs: searching for magic bullets
}

\author{
Luis Adrián Rosales-Hernández, Francisco Eduardo Rivas-Larrauri, Marco Antonio Yamazaki-Nakashimada \\ Servicio de Inmunología. Instituto Nacional de Pediatría, SSA. Ciudad de México, México.
}

\begin{abstract}
RESUMEN
La autoinmunidad y la inmunodeficiencia eran consideradas previamente condiciones mutuamente excluyentes, sin embargo, con el avance en el entendimiento de los mecanismos reguladores y del análisis genético se ha encontrado que la relación es más estrecha de lo previsto. Un paciente puede presentar manifestaciones infecciosas y autoinmunes simultáneamente. Así, existen medicamentos polifacéticos (llamados «balas mágicas» por Paul Ehrlich) que han demostrado propiedades inmunomoduladoras y antimicrobianas que pueden ser de utilidad. En este artículo se revisan los mecanismos antiinfecciosos de fármacos utilizados rutinariamente como inmunosupresores; además de las propiedades inmunomoduladoras de antibióticos, antiparasitarios, reguladores de las vías metabólicas e inhibidores de la angiogénesis que pudiesen tener implicaciones clínicas.
\end{abstract}

Palabras clave: Inmunidad, autoinmunidad, inmunomodulación, antimicrobianos.

\author{
Abreviaturas: \\ $\mathrm{CMV}=$ citomegalovirus \\ $\mathrm{DHODH}=$ dihidroorotato deshidrogenasa, \\ IFN- $\alpha \beta=$ interferón alfa-beta, \\ IMPDH = inosina-monofosfato deshidrogenasa, \\ LFA-1 = antígeno-1 asociado a la función del linfocito, \\ MHC-II = complejo principal de histocompatibilidad clase II, \\ NFAT = factor nuclear asociado a células T activadas; \\ $\mathrm{NF}-\kappa \mathrm{B}=$ factor nuclear $\kappa \mathrm{B}, \mathrm{TCR}=$ receptor del linfocito $\mathrm{T}$, \\ TLR $=$ receptor tipo Toll, \\ TNF- $\alpha=$ factor de necrosis tumoral alfa, \\ $\mathrm{VEFG}=$ factor de crecimiento endotelial vascular, \\ $\mathrm{VHC}=$ virus de la hepatitis $\mathrm{C}$.
}

Simbología: líneas rojas: inhibición/bloqueo; frases entre corchetes: efecto del medicamento.

Financiamiento:

Conflicto de intereses: El Dr. Marco Antonio Yamazaki-Nakashimada ha recibido honorarios como ponente de los laboratorios Octapharma $A^{\circledR}{ }^{\circledR}$, Shire Pharmaceuticals México S.A. de C.V. y CSL Behring.

https://dx.doi.org/10.35366/92384

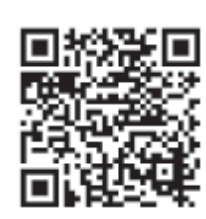

ABSTRACT

Autoimmunity and immunodeficiency were previously considered mutually exclusive conditions, however, with progress in understanding regulatory mechanisms and genetic analysis it has been found that the relationship is closer than predicted. A patient may present both infectious and autoimmune manifestations simultaneously. Thus, there are multifaceted drugs (called "magic bullets» by Paul Ehrlich) with immunomodulatory and antimicrobial properties. In this article, we review the anti-infective mechanisms of drugs routinely used as immunosuppressants; the immunomodulatory properties of antibiotics, antiparasitic, metabolic pathway regulators and angiogenesis inhibitors that may have clinical implications.

Keywords: Immunity, autoimmunity, immunomodulation, antimicrobial agents.

\section{INTRODUCCIÓN}

La autoinmunidad y la inmunodeficiencia eran consideradas previamente condiciones mutuamente excluyentes, sin embargo, con el avance en el entendimiento de los mecanismos reguladores y del análisis genético se ha encontrado que la relación es más compleja y estrecha de lo previsto. Un paciente puede presentar ambas manifestaciones; además, el tratamiento inmunosupresor en las enfermedades reumáticas genera frecuentemente complicaciones infecciosas, o viceversa, los procesos infecciosos activan una respuesta autoinmune. Es por ello que ante estos escenarios es necesario buscar agentes terapéuticos que tengan esa doble función: controlar el proceso infeccioso y abatir la respuesta autoinmune. ${ }^{1}$

Paul Ehrlich acuñó el término de «la bala mágica», la cual se refería a la búsqueda del medicamento ideal con propiedades antiinfecciosas sin causar daño, y lo logró con el «salvarsán», el primer medica- 
mento eficaz contra la sífilis. Así, el término original de «bala mágica» conlleva propiedades antiinfecciosas. ${ }^{2}$

En este artículo revisamos aquellos medicamentos polifacéticos que poseen propiedades antiinfecciosas e inmunomoduladoras que son utilizados con mayor frecuencia en el tratamiento del paciente con autoinmunidad. Es importante destacar que, si bien estos medicamentos poseen ambas propiedades, en la práctica no siempre se utilizan con ambos fines (Figura 1 y Tabla 1).

\section{Medicamentos predominantemente inmunomoduladores con propiedades antimicrobianas}

Ciclosporina A. Los inhibidores de la calcineurina se han utilizado en el tratamiento inmunosupresor de pacientes trasplantados, como alternativa terapéutica en enfermedades autoinmunes refractarias y en el síndrome hemofagocítico. Dentro de este grupo de medicamentos destaca la ciclosporina $A$ (CsA). El medicamento modula las señales intracelulares producidas por la activación del receptor del linfocito-T bloqueando la actividad de la calcineurina. La calcineurina es una fosfatasa que en las células humanas regula la homeostasis intracelular del calcio (Ca2+), el metabolismo neuronal y la activación del linfocito T mediante el factor de transcripción NFAT; sin embargo, algunos hongos patógenos también poseen una calcineurina estructuralmente similar, por lo que son susceptibles a la inhibición de esta enzima. Los primeros estudios realizados para evaluar la virulencia de Candida albicans y hongos filamentosos como Aspergillus fumigatus

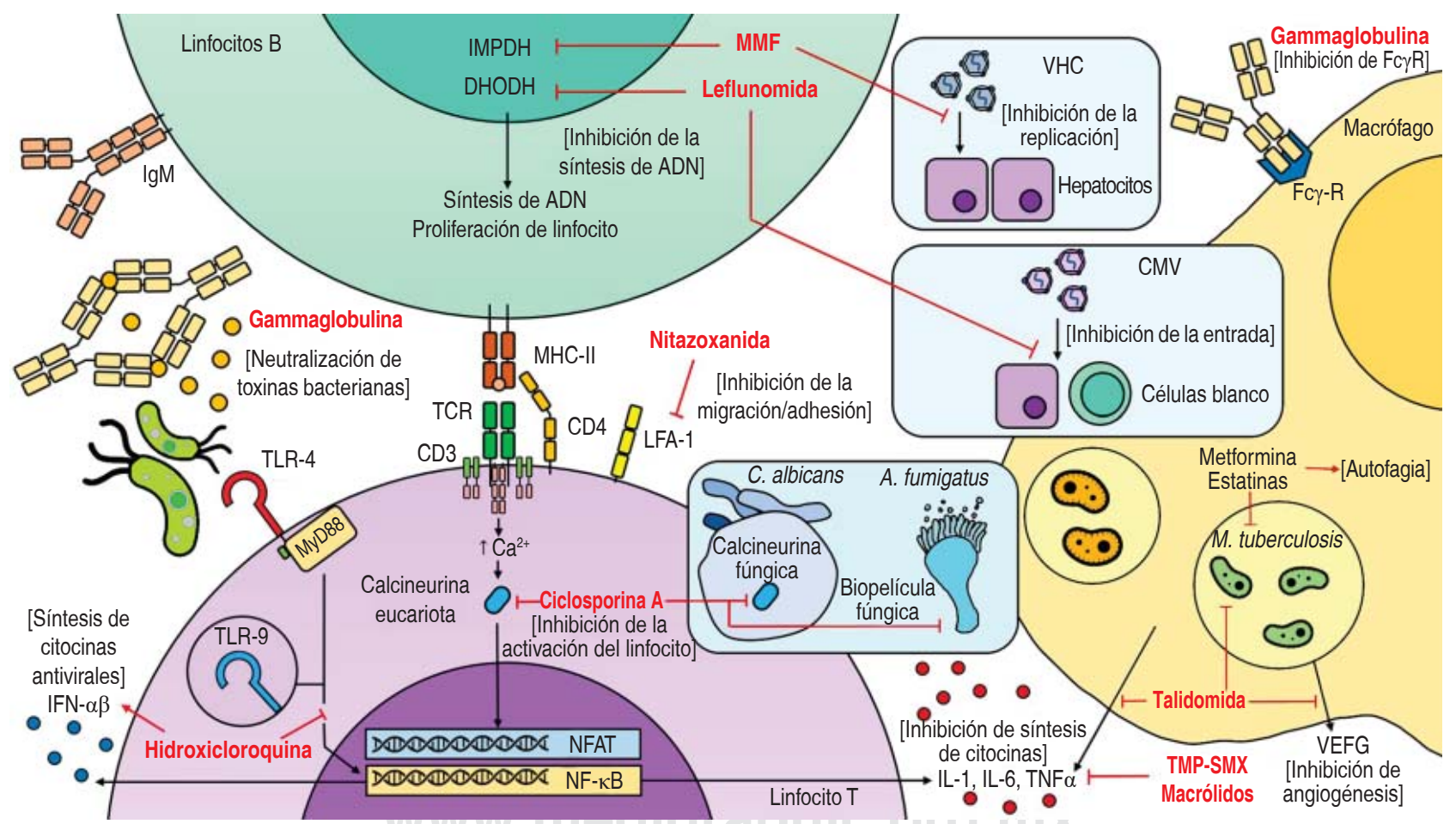

Figura 1: Medicamentos versátiles. La activación de la respuesta inmune adaptativa funciona como un modelo para explicar los mecanismos de acción de fármacos inmunosupresores con actividad antimicrobiana. Ciclosporina A inhibe la calcineurina fúngica y eucariota de hongos como Candida albicans y la biopelícula fúngica de Aspergillus fumigatus. Hidroxicloroquina, un antimalárico, actúa en la regulación de los TLR, con dos consecuencias fundamentales: inhibir la síntesis de citocinas proinflamatorias y favorecer un estado antiviral con la síntesis de interferones de tipo I. Talidomida, metformina y las estatinas son medicamentos que además de regular funciones metabólicas y de angiogenia, inhiben el crecimiento de micobacterias. Inmunosupresores como leflunomida y micofenolato de mofetilo son inhibidores de la síntesis de ADN con actividad antiviral. Algunos antibióticos y antiparasitarios como trimetoprim-sulfametoxazol, claritromicina y nitazoxanida también poseen propiedades inmunorreguladoras por distintos mecanismos. Finalmente, la gammaglobulina es el medicamento versátil con propiedades inmunomoduladoras y antiinfecciosas que puede ser utilizado en una amplia variedad de pacientes con autoinmunidad infectados, se muestra su efecto en la neutralización de toxinas bacterianas y la inhibición de los receptores de la porción Fc en la membrana del macrófago. 


\section{Tabla 1: Medicamentos predominantemente inmunosupresores con propiedades} antimicrobianas y reguladores de funciones metabólicas.

\begin{tabular}{|c|c|c|}
\hline Fármaco & Mecanismo inmunomodulador & Microorganismos susceptibles \\
\hline Ciclosporina & Inhibidor de la calcineurina & $\begin{array}{l}\text { Antifúngico: Candida albicans (efecto sinérgico con fluconazol) y espe- } \\
\text { cies de Candida no albicans, Aspergillus fumigatus, Aspergillus flavus, } \\
\text { Fusarium oxysporum, Coccidioides immitis, Histoplasma capsulatum, } \\
\text { Blastomyces dermatitidis, Schistosoma mansoni, Strongyloides spp., } \\
\text { Plasmodium falciparum, Leishmania major, Leishmania tropica, Toxoplas- } \\
\text { ma gondii } \\
\text { Antiviral: virus de la hepatitis C }\end{array}$ \\
\hline $\begin{array}{l}\text { Micofenolato de } \\
\text { mofetilo }\end{array}$ & $\begin{array}{l}\text { Inhibe la inosina-monofosfato } \\
\text { deshidrogenasa }\end{array}$ & $\begin{array}{l}\text { Antiviral: virus de la hepatitis C (sinergismo con ciclosporina A e interferón } \\
\text { alfa), influenza H5N1 (aviar), norovirus (sinergismo con ribavirina), Pneu- } \\
\text { mocystis jirovecii, virus de inmunodeficiencia humana, dengue, chikun- } \\
\text { gunya, hepatitis B, Coxsackie virus B3, virus del oeste del Nilo, virus de la } \\
\text { fiebre amarilla }\end{array}$ \\
\hline Leflunomida & $\begin{array}{l}\text { Inhibe la dihidroorotato } \\
\text { deshidrogenasa }\end{array}$ & Antiviral: citomegalovirus (resistente a ganciclovir), $\mathrm{VIH}$, dengue \\
\hline Hidroxicloroquina & $\begin{array}{l}\text { Inhibe la producción de citocinas } \\
\text { proinflamatorias a través de la } \\
\text { regulación negativa de TLR3, } \\
\text { TLR4 y TLR9 inactivando complejo } \\
\text { NF-kB - AP-1, disminuyendo la } \\
\text { producción de IL- } 1 \beta \text { y TNF- } \alpha \text {, de } \\
\text { autoanticuerpos y células NK }\end{array}$ & $\begin{array}{l}\text { Antiparasitario: Plasmodium spp. } \\
\text { Antiviral: virus de inmunodeficiencia humana, dengue, influenza, flavi- } \\
\text { virus, rubéola, virus de la hepatitis A, B y C, arenavirus, rabia, varicela } \\
\text { zóster, virus sincitial respiratorio, herpes simple, Epstein-Barr, poliovirus, } \\
\text { Newcastle virus } \\
\text { Antibacteriano: Coxiella burnetii (sinergismo con doxiciclina para fiebre } \\
\text { Q), Tropheryma whipplei, Legionella pneumophila, Francisella tularensis, } \\
\text { Mycobacterium tuberculosis, Mycobacterium avium, Salmonella Typhi, } \\
\text { Escherichia coli, Bacillus anthracis, Bacillus subtilis, Borrelia burgdorferi, } \\
\text { Brucella abortus, Staphylococcus aureus, Listeria monocytogenes } \\
\text { Antifúngico: Histoplasma capsulatum, Cryptococcus neoformans, Para- } \\
\text { coccidioides brasiliensis, Aspergillus fumigatus }\end{array}$ \\
\hline Talidomida & $\begin{array}{l}\text { Inhibición de TNF- } \alpha \text { y del VEGF } \\
\text { (angiogénesis) }\end{array}$ & Antimicobacteria: Mycobacterium tuberculosis y Mycobacterium leprae \\
\hline Estatinas & $\begin{array}{l}\text { Inhibición de la HMG-CoA } \\
\text { reductasa, inhibición de molécu- } \\
\text { las de adhesión de la respuesta } \\
\text { inflamatoria }\end{array}$ & $\begin{array}{l}\text { Antimicobacteria: Mycobacterium tuberculosis y Mycobacterium leprae } \\
\text { Antibacteriano: Borrelia burgdorferi, Staphylococcus aureus, Enterococ- } \\
\text { cus spp., Acinetobacter baumannii, Staphylococcus epidermidis, Entero- } \\
\text { bacter aerogenes, Escherichia coli, Proteus mirabilis, Enterobacter cloacae } \\
\text { Antiviral: influenza virus (H5N1, H1N1) }\end{array}$ \\
\hline Metformina & Antineoplásico inmunorregulador & Antimicobacteria: Mycobacterium tuberculosis \\
\hline Pioglitazona & $\begin{array}{l}\text { Restaura especies reactivas de } \\
\text { oxígeno de origen mitocondrial. } \\
\text { Potencia estallido respiratorio en la } \\
\text { enfermedad granulomatosa crónica }\end{array}$ & $\begin{array}{l}\text { Antibacteriano: Candida albicans, Streptococcus pneumoniae, Escheri- } \\
\text { chia coli, Klebsiella pneumoniae, Staphylococcus aureus }\end{array}$ \\
\hline
\end{tabular}

demostraron un papel importante de la calcineurina en el metabolismo fúngico, incluyendo morfogénesis, crecimiento y virulencia. ${ }^{3}$

La inhibición de la calcineurina en las células eucariotas condiciona la disminución de la transcripción de los genes que codifican la interleucina-2 (IL-2), factor de necrosis tumoral alfa (TNF- $\alpha$ ), IL-3, factor estimulante de colonias de granulocitos y monocitos (GM-CSF) entre otras citocinas, lo cual justifica en gran medida su efecto antiinflamatorio. ${ }^{4}$

La CsA actúa de forma sinérgica con otros medicamentos antifúngicos en microorganismos susceptibles: 1) incrementa la sensibilidad a azoles en modelos de Candida albicans, a través de la inhibición de la biopelícula fúngica (biofilm); ${ }^{5}$ 2) in vitro potencia los efectos de la equinocandina y caspofungina contra Aspergillus fumigatus; el 
incremento en las concentraciones de caspofungina produce un «efecto de crecimiento reverso", debido a que la inhibición de la calcineurina disminuye los niveles de $\beta$-1-3-glucanos, produciendo un incremento compensatorio de la síntesis quitina. Esta respuesta de «estrés» en la pared celular de hongos filamentosos es susceptible al bloqueo terapéutico con inhibidores de $\beta$-1-3-glucanos, de la síntesis de quitina y de la calcineurina; $;, 73$ ) incrementa la actividad de anfotericina $B$, azoles y caspofungina contra la plantónica y la biopelícula de $C$. parapsilosis; $^{8} 4$ ) se ha demostrado un efecto sinérgico con equinocandina y azoles (modelo con Candida albicans) y caspofungina (modelo de Aspergillus fumigatus) mediado por la inhibición de la proteína Hsp-90. ${ }^{9}$ Por otro lado, CsA interfiere en el proceso de presentación del antígeno, inhibe la síntesis de IL-17 por las células Th17 así como la producción de autoanticuerpos dependiente de los linfocitos-T, por lo que el mecanismo antifúngico de este medicamento se basa de manera casi exclusiva en la actividad que ejerce directamente sobre el hongo. ${ }^{10}$

Hidroxicloroquina. La hidroxicloroquina (HCQ) es un derivado de la cloroquina, en la que un grupo $\mathrm{N}$-etilo ha sido beta-hidroxilado. Su efecto contra parásitos del complejo Plasmodium spp es dependiente de la sensibilidad a este medicamento en distintas regiones endémicas del mundo. La utilidad de este medicamento en enfermedades autoinmunes es bien conocida. ${ }^{11}$ Su espectro inmunomodulador se basa en la capacidad de suprimir la producción de citocinas proinflamatorias a través de la regulación negativa de los receptores de tipo Toll (TRL), inactiva el complejo NF-kB-AP-1, disminuye la producción de IL-1 $\beta$ y TNF- $\alpha$, de autoanticuerpos y de células NK, ${ }^{12}$ interfiere con la presentación antigénica y la síntesis de ARN y ADN. ${ }^{13}$ El primer modelo en describir sus efectos antimicrobianos se basó en la determinación del $\mathrm{pH}$ en las vacuolas de replicación de Coxiella burnetii, agente causal de la fiebre $\mathrm{Q} .{ }^{11}$

La HCQ es una base débil que se acumula en el medio ácido de los organelos intracelulares e inhibe la replicación de diferentes tipos de virus interfiriendo en el tráfico lisosomal, endosomal o en el proceso de maduración de los viriones. ${ }^{14} \mathrm{El}$ incremento del $\mathrm{pH}$ en las vesículas intracelulares se correlaciona con su actividad contra microorganismos diversos en estudios in vitro: Staphylococcus aureus, Legionella pneumophila, Francisella tularensis, Mycobacterium tuberculosis, Salmonella enterica serovar Typhi, entre otros. ${ }^{11,15,16}$
La cloroquina y la $\mathrm{HCQ}$ disminuyen la expresión de la glucoproteína 120 (gp120) de los viriones procedentes de células infectadas por el $\mathrm{VIH}-1$, se relacionan con la interferencia de la glucosilación de las proteínas virales y el incremento del $\mathrm{pH}$ en los endosomas que participan en la expresión de gp120. ${ }^{17,18} \mathrm{La}$ adición de HCQ al tratamiento antirretroviral permite alcanzar una cuenta de linfocitos TCD4+ superiores en menor tiempo en los pacientes infectados con $\mathrm{VIH} .{ }^{12}$ Sin embargo, aquéllos sin tratamiento antirretroviral que reciben solo $\mathrm{HCQ}$ demostraron tener cuentas de linfocitos TCD4+ menores al grupo placebo, con incremento en la replicación viral. ${ }^{19}$ Así, su utilidad en la clínica debe ser evaluada con detalle.

Se ha demostrado la capacidad de la HCQ de inhibir los cuatro serotipos del virus del dengue (DENV1-4), además de inducir citocinas como IL-6, IL-12 p19 e IL-12 p40. ${ }^{14}$ Las respuestas antivirales mediadas tanto por la inmunidad innata como adaptativa son eficientes contra virus hepatotropos y respiratorios estacionales. ${ }^{20}$

En la cohorte estudiada por Feldman y colaboradores, los pacientes con lupus eritematoso sistémico tratados con hidroxicloroquina mostraron un riesgo reducido de infecciones comparado con aquéllos que nunca la utilizaron. La adición de hidroxicloroquina al tratamiento de las vasculitis ANCA positivas disminuyen la actividad de la enfermedad, mejoran la calidad y la expectativa de vida. ${ }^{21}$ En micología, la $\mathrm{HCQ}$ produce la deprivación de hierro dependiente del $\mathrm{pH}$ y favorece la fusión del fagolisosoma en células infectadas por hongos patógenos como Histoplasma capsulatum, Aspergillus nidulans y Cryptococcus neoformans en modelos in vitro. ${ }^{11}$ Un reporte de onicomicosis crónica por Aspergillus niger demostró mejoría clínica significativa con dosis de 200 mg/día en adultos. ${ }^{22}$

Leflunomida. La leflunomida es un profármaco con actividad inmunomoduladora mediada por la inhibición de la síntesis de pirimidinas, utilizado en el tratamiento de la artritis reumatoide y en la prevención del rechazo de trasplantes. Inhibe la enzima dihidroorotato deshidrogenasa (DHODH) y disminuye la actividad de tirosina-cinasas, llevando a las células $T$ y $B$ a una fase $S$ del ciclo celular y activando vías apoptóticas a través de $552 .{ }^{23}$ Sus efectos antiinflamatorios se explican por la disminución en la expresión de moléculas de adhesión en el endotelio, supresión del factor de crecimiento endotelial vascular (VEGF), aumento de citocinas antiinflamatorias como IL-10, bloqueo del NF-kB y un 
cambio en la polaridad de la respuesta inmune, de un perfil Th1 a Th2, por lo que es de utilidad en la artritis reumatoide. ${ }^{24}$

La leflunomida actúa en el ensamblaje de la cápside viral, no en la replicación del ADN y por lo tanto, no existe resistencia cruzada con otros fármacos activos contra el citomegalovirus (CMV). De los pacientes trasplantados con infección por CMV refractaria a los que se agregó leflunomida, en $53 \%$ se reportaron cargas indetectables a largo plazo, lo que demostró ser una herramienta útil en casos seleccionados, incluyendo aquéllos en los que se encuentra resistencia a ganciclovir. ${ }^{25}$

En 1999 se describió por primera vez las propiedades antivirales de leflunomida en modelos in vitro de células infectadas por CVM, virus del herpes simple (VHS) y poliomavirus $\mathrm{BK} ;{ }^{26}$ sin embargo, en un estudio en 2018 no encontró correlación entre las cargas virales de pacientes con nefropatía por poliomavirus VK y las concentraciones de leflunomida como tratamiento adyuvante. ${ }^{27}$

Micofenolato de mofetilo. El micofenolato de mofetilo (MMF) actúa como un potente supresor de la proliferación de linfocitos que inhibe a la enzima inosina-monofosfato deshidrogenasa (IMPDH), utilizado inicialmente en la prevención del rechazo de aloinjertos; posteriormente se documentó su utilidad en el tratamiento de enfermedades autoinmunes. ${ }^{28}$ Los efectos inmunosupresores del MMF son potentes y su utilidad como agente antimicrobiano adyuvante aún es controvertida; a continuación, se mencionan las propiedades de este fármaco demostradas principalmente en modelos in vitro.

De forma interesante, el MMF inhibe la replicación del virus de la hepatitis $\mathrm{C}$ (VHC) a través de la supresión de autofagia en células hepáticas; ${ }^{29}$ este efecto citotóxico se observa a dosis de entre $1-5 \mu \mathrm{g} / \mathrm{mL}$, una dosis menor a $10 \mu \mathrm{g} / \mathrm{mL}$ que es la considerada inmunosupresora. El MMF y la ribavirina incrementan la síntesis de interferones de tipo 1 (IFN- $\alpha \beta$ ) a través de genes inductores de interferones (interferonstimulated gene; ISG) como IRF-1, un mecanismo independiente de la inhibición de la IMPDH, lo que favorece un estado antiviral. ${ }^{30,31}$ El ARNm del virus de la influenza aviar H5N1 también se ve afectado con la administración de este medicamento, disminuyendo su replicación en modelos in vitro. ${ }^{32} \mathrm{EI} \mathrm{MMF}$ no inhibe la replicación del virus del herpes simple $1-2$, ni el virus de la varicela zóster (VVZ), pero sí ha demostrado un efecto contra el CMV, potenciando el efecto del ganciclovir in vivo e in vitro. ${ }^{33}$
Siebert aisló 16 compuestos derivados del ácido micofenólico con propiedades antimicrobianas contra cepas de Klebsiella pneumoniae, Escherichia coli, Pseudomonas aeruginosa y Staphylococcus aureus en diferentes líneas celulares. ${ }^{34}$ La depleción de trifosfato de guanosina en linfocitos- $T$ inhibe la actividad de la transcriptasa inversa y disminuye la expresión de gp120 en células infectadas por el VIH. Reportes aislados confieren al MMF un efecto protector contra infecciones causadas por Pneumocystis jirovecii, virus Coxsakie, virus del oeste del Nilo, virus de la fiebre amarilla y norovirus..$^{28,35}$ Se trata de un medicamento con un potente efecto inmunosupresor; sin embargo, se requieren más estudios para evaluar su eficacia en pacientes con autoinmunidad e infección concomitante.

Talidomida. La talidomida se introdujo al mercado en 1956 como un auxiliar en la conciliación del sueño que posteriormente se volvió popular en el tratamiento de la hiperémesis gravídica. Los reportes de amelia y focomelia en décadas posteriores forzaron su retiro del mercado. En la actualidad se encuentra aprobada para el tratamiento del eritema nodoso y el mieloma múltiple. ${ }^{36}$ En el contexto de las enfermedades autoinmunes la talidomida cobra un papel importante por sus características inmunosupresoras y antiangiogénicas: 1) acelera la degradación del ARNm del factor de necrosis tumoral alfa (TNF- $\alpha$ ), ya que disminuye su producción en las células mononucleares; ${ }^{37} 2$ ) inhibe la producción de IL-12 mediada por NF- $\kappa B ;{ }^{38} 3$ ) incrementa la expresión de IL-4 e IL-10, lo que favorece la polarización de la respuesta a un perfil Th2; 4) inhibe la síntesis del factor de crecimiento endotelial vascular en las células endoteliales e impide la angiogénesis; y 5) produce la regulación negativa en la expresión de moléculas de adhesión. ${ }^{39}$ Además, favorece la activación de los linfocitos TCD8+ más que los TCD4+, debido al incremento en la expresión de interferón gamma (IFN- $\gamma$ ), citocina importante en la activación de la sintasa inducible de óxido nítrico, fundamental en la liberación de especies reactivas de nitrógeno. ${ }^{36,40}$

La talidomida se ha evaluado como un agente adyuvante en el tratamiento de la infección por Mycobacterium tuberculosis y Mycobacterium leprae. En este contexto, el efecto más importante es la supresión del TNF- $\alpha$ por los macrófagos, ya que reducen la respuesta inflamatoria y elevan los niveles de IFN- $\gamma$, IL-2 e IL-12, lo que favorece la activación del estallido respiratorio..$^{40}$ Los pacientes con tuber- 
culosis pulmonar han mostrado ganancia ponderal durante el tratamiento con talidomida; observación que también ocurre en la coinfección con el $\mathrm{VIH}^{41}$ Existen circunstancias en las que los pacientes con infección por micobacterias presentan una exacerbación de su patología autoinmune, escenario en el que la talidomida tiene un mayor potencial y cuya utilidad debería ser considerada. Además, este medicamento se ha utilizado como tratamiento adyuvante en casos de meningitis tuberculosa, tuberculomas, ${ }^{42}$ colitis en la enfermedad granulomatosa crónica ${ }^{43}$ y enfermedad de Crohn. ${ }^{39}$

\section{Medicamentos predominantemente antimicrobianos con propiedades inmunomoduladoras}

Los antibióticos han sido utilizados en el tratamiento de las enfermedades autoinmunes con el fundamento que las infecciones son potenciales «disparadoras» de estas entidades. Ejemplos en que se ha asociado la presencia de microorganismos bien identificados en enfermedades autoinmunes con tratamiento antibiótico son diversos: macrólidos, tetraciclinas y fluoroquinolonas para artritis reumatoide, ciprofloxacino para artritis reactiva crónica, trimetoprim-sulfametoxazol como protocolo en granulomatosis con poliangeítis, erradicación del $H$. pylori en púrpura trombocitopénica crónica y tratamiento antimicrobiano empírico en pacientes con síndrome de anticuerpos antifosfolípidos. ${ }^{44}$

En esta sección se discuten los mecanismos de algunos agentes antimicrobianos usados para modificar el curso de las enfermedades autoinmunes.

Claritromicina. Los macrólidos han sido ampliamente utilizados en el tratamiento de enfermedades respiratorias y digestivas infecciosas. Son agentes bacteriostáticos que inhiben la síntesis de proteínas al unirse reversiblemente a la subunidad ribosomal $50 \mathrm{~s} .{ }^{45}$

Los macrólidos poseen un espectro antiinflamatorio e inmunomodulador que ha sido probado en modelos de enfermedades inflamatorias crónicas como la panbronquiolitis difusa, enfermedad pulmonar obstructiva crónica, fibrosis quística $(F Q)^{46}$ y bronquiectasias no-FQ. ${ }^{47}$ La claritromicina, azitromicina y telitromicina inhiben procesos inflamatorios agudos en modelos de sepsis inducida por lipopolisacárido, al suprimir la síntesis de citocinas proinflamatorias como TNF- $\alpha$ e IL- $1 \alpha$ mediante la inhibición de la vía de NF- $\kappa B$ en las células mononucleares. ${ }^{48-50}$ Se ha documentado que la azitromicina inhibe la prolife- ración de los linfocitos-T a través de la vía m-TOR (target of rapamycin). ${ }^{51}$ Cabe destacar que el FK-506 y la rapamicina son estructuralmente similares a los macrólidos y poseen la capacidad de modular la activación del linfocito T a través de la calcineurina. Otros efectos que se han demostrado son el incremento en la degranulación de los neutrófilos, la atenuación del estallido respiratorio y de la expresión de moléculas de adhesión. ${ }^{52}$

La artritis reumatoide y la enfermedad de Still del adulto representan un grupo de enfermedades para los que la claritromicina ha demostrado disminuir el tiempo de inducción a la remisión en sinergismo con los fármacos modificadores de la enfermedad..$^{53}$ Casos de artritis reumatoide refractaria al tratamiento inmunosupresor han mejorado cuando se agrega claritromicina como adyuvante, ya que inhiben la síntesis de fosfolipasa A2 y prostaglandina E2. En enfermedad de Still del adulto se asoció claritromicina con metotrexato y glucocorticoides, lo cual permitió la disminución en el uso de esteroides..$^{54,55}$ Una hipótesis sustenta que la periodontitis producida por bacterias anaeróbicas es importante en la artritis reumatoide; sin embargo, la atribución de una causa estrictamente infecciosa a una enfermedad autoinmune aún es controversial. ${ }^{56}$

Recientemente se publicó la eficacia de la claritromicina junto con la inmunoglobulina endovenosa en el tratamiento de la enfermedad de Kawasaki. Moléculas como FK-565 tienen similitud estructural con los patrones moleculares asociados con microbios (MAMP's) y poseen tropismo para desarrollar una respuesta inflamatoria en los vasos sanguíneos, incluyendo arterias coronarias. Dentro de los MAMP's con estas propiedades se encuentran aquéllos que derivan de Yersinia pseudotuberculosis y otras aerobacterias. Otros reportes describen la asociación de Mycoplasma pneumoniae con la enfermedad de Kawasaki, en la que la claritromicina tiene un papel terapéutico primario. A pesar de que el uso concomitante de claritromicina no afecta la duración de la fiebre, sí disminuye el riesgo de recaída y el periodo de hospitalización. ${ }^{57}$

Nitazoxanida. La nitazoxanida es un antiparasitario de amplio espectro. Inicialmente fue aprobado por la Food and Drug Administration (FDA) para infecciones por Cryptosporidium parvum y Giardia lamblia:;8 hoy se reconocen nuevos alcances de su cobertura antimicrobiana.

La nitazoxanida actúa contra el virus de la influenza al bloquear la maduración de la hemaglutinina 
en un estado postraduccional, sin tener efectos en la neuraminidasa. En las células mononucleares estimula la síntesis de interferones de tipo I para inducir un estado antiviral. ${ }^{59}$ Posee la capacidad de inhibir a la proteína CHIK 26s, bloqueando la fusión a la membrana de las células hospederas en la infección por el virus del chikungunya. La nitazoxanida se considera un antiviral de espectro extendido que puede ser utilizado en la infección por virus del dengue, fiebre amarilla, virus sincitial respiratorio, parainfluenza, coronavirus, rotavirus, norovirus, virus $\mathrm{JC}$, virus de la hepatitis $\mathrm{B}$, hepatitis $\mathrm{C}$, $\mathrm{VIH}^{59,60}$ y Zika. ${ }^{61} \mathrm{~A}$ pesar de los efectos in vitro contra el virus de la hepatitis $C$, no se ha demostrado que la nitazoxanida mejore la morbimortalidad relacionada con esta infección. ${ }^{62}$

Además de su actividad antiparasitaria, la nitazoxanida demuestra actividad contra bacterias aerobias, grampositivas y gramnegativas, así como cepas del complejo Mycobacterium tuberculosis y Mycobacterium leprae al inducir el proceso de autofagia en las células del sistema fagocítico mononuclear y la inhibición de la enzima la piruvato deshidrogenasa de Escherichia coli. ${ }^{63}$

Posee papel en la modulación de carcinogénesis y se han demostrado estas propiedades: inhibe el crecimiento y la progresión de células tumorales mediante la glutatión-S-transferasa P1, induce autofagia en las células tumorales, inhibe la síntesis de citocinas y suprime la actividad del oncogén c-Myc. ${ }^{64}$ Se ha propuesto su papel como un inhibidor de la molécula PD-1 en el proceso de inmunovigilancia contra las células tumorales en un modelo de cáncer de ovario. ${ }^{65}$

Trimetoprim-sulfametoxazol (TMP-SMX). Trimetoprim inhibe la enzima dihidrofolato reductasa bacteriana (con mayor preferencia que a la humana); el sulfametoxazol es un análogo estructural del ácido paraaminobenzoico (PABA) que inhibe a la enzima dihidropteroato reductasa, el cual disminuye la formación del ácido dihidrofólico, un intermediario en la formación de tetrahidrofolato (THF). La reducción de la cantidad del THF inhibe la síntesis de timidina y por ende, la síntesis del ADN. ${ }^{66}$ La utilidad del TMPSMX se ha evaluado en pacientes con vasculitis.

La granulomatosis con poliangeítis (GPA) es una vasculitis de pequeños vasos que se caracteriza por la presencia de anticuerpos anticitoplasma de neutrófilos (ANCAs), en la cual el tratamiento con esteroides y ciclofosfamida ha permitido el control de la enfermedad; sin embargo, su asociación con infecciones de repetición de la vía aérea superior llevó a Stegeman y a sus colegas a realizar un estudio controlado aleatorizado en el que se demostró la eficacia del TMP-SMX en la reducción de recaídas cuando se usaba como profilaxis junto con el tratamiento inmunosupresor. ${ }^{67}$ Se ha documentado una asociación entre portadores crónicos de Staphylococcus aureus y un incremento en las recaídas de GPA, por lo que se postula que TMPSMX suprime la replicación bacteriana en el tracto respiratorio superior, disminuyendo la actividad de la enfermedad mediada por su «disparador». Los títulos de ANCAs en los pacientes tratados con TMP-SMX disminuyeron con tratamiento profiláctico. ${ }^{68}$

\section{MINOCICLINA}

La minociclina es una tetraciclina semisintética de segunda generación con propiedades antibióticas contra bacterias grampositivas y gramnegativas, con uso principalmente en acné vulgaris y enfermedades de transmisión sexual. ${ }^{68-71}$ Sin embargo, se han documentado propiedades antiinflamatorias y antiapoptóticas que inhiben proteólisis, angiogénesis y metástasis tumorales. ${ }^{68}$ Reduce la quimiotaxis de neutrófilos, quela el calcio inhibiendo la activación de los linfocitos TCD4 y posee actividad anticolagenasa, por lo que se ha investigado su utilidad en artritis reumatoide, pioderma gangrenoso, esclerodermia y enfermedad inflamatoria intestinal. Particularmente por sus propiedades lipofílicas con alta penetración en el sistema nervioso central (SNC) con efecto mitigante en la expresión de citocinas inflamatorias en SNC y activación de la microglía se ha estudiado en esclerosis múltiple con efecto neuroprotector. ${ }^{70,71}$

\section{MEDICAMENTOS CON FUNCIÓN ANTIMICROBIANA E INMUNOMODULADORA}

\section{Gammaglobulina}

Tiene como peculiaridad una función equilibrada antiinfecciosa e inmunomoduladora. La inmunoglobulina policlonal o estándar consiste en una preparación de inmunoglobulinas (la mayoría IgG) purificadas de plasma humano. El tratamiento con inmunoglobulina endovenosa (IGIV) o subcutánea (IGSC) se ha convertido en parte esencial del tratamiento de una gran variedad de inmunodeficiencias primarias y enfermedades autoinmunes. ${ }^{72,73}$ El tratamiento 
con inmunoglobulina históricamente se empezó a utilizar para el reemplazo terapéutico en pacientes con inmunodeficiencias. ${ }^{74}$

Fue en 1981 cuando en un paciente con inmunodeficiencia primaria (hipogammaglobulinemia) y autoinmunidad (púrpura trombocitopénica autoinmune) se demostró sus propiedades inmunomoduladoras. ${ }^{75,76}$

Actividad antígeno específica: se basa en el concepto de que contiene una alta concentración de IgG con capacidad de opsonización, neutralización y activación de complemento. ${ }^{72}$ Acción inmunomoduladora: son múltiples mecanismos de acción implicados en la patología autoinmune e inflamatoria; bloquea de forma transitoria los receptores $\mathrm{Fc} \gamma$ de los macrófagos, restaura la red idiotipo-antiidiotipo, la regulación a la baja de receptores $\mathrm{Fc} \gamma$, induce la proliferación de linfocitos TCD8+ supresores, neutraliza toxinas bacterianas que actúan como superantígenos, modula la producción de citocinas como IL-1, IL-6 y TNF, entre otros. ${ }^{72,76,77}$

\section{MEDICAMENTOS QUE REGULAN FUNCIONES METABÓLICAS CON PROPIEDADES ANTIMICROBIANAS E INMUNOMODULADORAS}

\section{Metformina}

Además de ser el antidiabético de elección en el tratamiento inicial de la diabetes mellitus tipo 2, la metformina posee propiedades en la regulación de la respuesta inflamatoria y en la inhibición de la carcinogénesis. Los mecanismos de acción son múltiples y complejos: inhibe la enzima glicerofosfato deshidrogenasa y activa la 5'-proteincinasa activada por AMP cíclico (AMPK) en los hepatocitos, inhibe al complejo I de la cadena respiratoria mitocondrial disminuyendo los requerimientos energéticos de cada célula y regula negativamente a la proteína LKB1 suprimiendo el ciclo celular. Los efectos terapéuticos resultan en la disminución de la producción hepática de glucosa (gluconeogénesis), el incremento de la sensibilidad de los tejidos periféricos a la insulina y disminución de la lipogénesis. ${ }^{78}$ En estudios experimentales, la regulación en la fosforilación de AMPK a través de la vía mTOR-STAT3 disminuye la activación de linfocitos-B y la producción de autoanticuerpos en modelos murinos de lupus eritematoso sistémico..$^{79,80}$

La metformina se ha planteado como un importante agente adyuvante en el tratamiento de infecciones por micobacterias en pacientes diabéticos y no diabéticos: induce la formación de especies reactivas de oxígeno y nitrógeno en las células infectadas por Mycobacterium tuberculosis, favorece la autofagia de los macrófagos y la síntesis de IFN- $\gamma$, lo que induce una respuesta Th1, ${ }^{81}$ potencia la acción de los medicamentos antifímicos convencionales, disminuye la carga de bacilos ${ }^{82}$ y el tiempo de negativización de cultivos de pacientes con tuberculosis y cavitaciones después de dos meses de tratamiento. ${ }^{83}$

La modulación terapéutica de los mecanismos del hospedero para erradicar las infecciones constituye un nuevo paradigma en el descubrimiento de nuevos medicamentos. La respuesta innata contra el complejo Mycobacterium tuberculosis incluye la activación del estallido respiratorio por las especies reactivas de oxígeno (ERO) y nitrógeno (ERN) y la activación de la maquinaria en los fagosomas que promueve la autofagia. La autofagia es controlada por la vía m-TOR y el complejo AMPK. La activación de la vía AMPK mediada por metformina inhibe el crecimiento intracelular de Mycobacterium tuberculosis con dos consecuencias fundamentales: evitar el progreso de la enfermedad y potenciar la eficacia de los antifímicos convencionales. Los efectos protectores de la metformina son: incrementar la producción de ERO en los macrófagos y disminuir el pH en los fagosomas donde se encuentran las micobacterias. Además, los linfocitos TCD8+ incrementan la producción de interferón gamma (IFN- $\gamma$ ) en ratones no infectados, lo que indica que la metformina tiene un impacto en la inmunidad pulmonar independientemente del estado infeccioso. ${ }^{58}$ La adición como esquema normoglucemiante en pacientes con DM2 infectados con tuberculosis es recomendable. ${ }^{84}$

Estatinas. Las estatinas son inhibidores competitivos de la hidroximetilglutaril-CoA reductasa (HMGCoA reductasa) interfiriendo en la conversión de HMG-CoA a mevalonato; por lo tanto, interfieren en una reacción temprana de la síntesis de colesterol. Los efectos cardioprotectores se traducen en una disminución del colesterol sérico a expensas de lipoproteínas de baja densidad (LDL). Los efectos pleiotrópicos de las estatinas son múltiples. ${ }^{85}$

Las estatinas son medicamentos utilizados en el tratamiento de la enfermedad cardiovascular producida por la formación de placas de ateroma. Son análogos estructurales del mevalonato que tienen la capacidad de inhibir a la HMG-CoA reductasa, que en los mamíferos es responsable de la síntesis del colesterol. Ampliamente usadas en el tratamiento de 
las dislipidemias, las estatinas ahora se reconocen como medicamentos inmunomoduladores con importantes efectos pleiotrópicos. En un modelo de Mycobacterium leprae se encontró que los niveles de estatinas no alteran la invasión de las micobacterias, pero sí incrementan la permeabilidad de la membrana, lo cual se relaciona con un efecto sinérgico con la rifampicina. ${ }^{82}$ Además, se ha documentado su utilidad en la infección por el virus de la influenza por mecanismos relacionados con la inducción de la autofagia. ${ }^{86}$

Atorvastatina y simvastatina muestran la capacidad de inhibir el crecimiento de cepas de Staphylococcus aureus resistente a meticilina, Enterococcus resistente a vancomicina, Acinetobacter baumannii, entre otros. En las bacterias, la HMG-CoA reductasa es fundamental para la biosíntesis de isoprenos; sin embargo, su afinidad a las estatinas es 10,000 veces más débil que en las células eucariotas; por ello, el mecanismo microbiocida se atribuye a otras propiedades, como efectos citotóxicos, supresión del crecimiento celular e incremento en la apoptosis. ${ }^{87-89}$ Dentro de la respuesta inflamatoria las estatinas disminuyen los niveles del antígeno asociado con función del linfocito-1 (LFA-1) y por ende, la adhesión leucocitaria y el proceso de homing en los ganglios linfáticos periféricos. ${ }^{90,91}$

Tiazolidinedionas. Los agonistas del receptor de peroxisomas PPAR $\gamma$ y PPAR $\alpha$ son medicamentos que incrementan la sensibilidad a la insulina, disminuyen la producción hepática de glucosa y favorecen la movilización de lípidos en los pacientes diabéticos. Estos efectos se han asociado con un incremento en la defensa contra microorganismos como Candida albicans, Staphylococcus aureus, Klebsiella pneumoniae y Streptococcus pneumoniae. Uno de los mecanismos propuestos es el incremento de las especies reactivas de oxígeno de origen mitocondrial ( $m t-E R O)$ en ratones con enfermedad granulomatosa crónica con defecto en gp91phox, incrementando la función de microbiocida de las células mononucleares en infecciones por S. aureus. ${ }^{92}$

\section{CONCLUSIONES}

Existen medicamentos con múltiples mecanismos biológicos y poseen efectos antimicrobianos e inmunomoduladores, sin embargo, son necesarios mayores estudios para poder concluir su utilidad clínica. La idea de Paul Ehrlich de encontrar agentes terapéuticos ideales (balas mágicas) continúa vigente a más de 100 años de su concepción.

\section{REFERENCIAS}

1. Schmidt RE, Grimbacher B, Witte T. Autoimmunity and primary immunodeficiency: two sides of the same coin? Nat Rev Rheumatol. 2018; 14 (1): 7-18. doi: 10.1038/ nrrheum.2017.198.

2. García-Sánchez JE, García E, Lucila-Merino M. Cien años de la bala mágica del Dr. Ehrlich (1909-2009). Enferm Infecc Microbiol Clin. 2010; 28 (8): 521-533. doi: 10.1016/j. eimc.2009.07.009.

3. Juvvadi PR, Lee SC, Heitman J, Steinbach WJ. Calcineurin in fungal virulence and drug resistance: prospects for harnessing targeted inhibition of calcineurin for an antifungal therapeutic approach. Virulence. 2017; 8 (2): 186-197. doi: 10.1080/21505594.2016.1201250.

4. Ishida $\mathrm{Y}$, Matsuda H, Kida K. Effect of cyclosporin A on human bone marrow granulocyte- macrophage progenitors with anti-cancer agents. Acta Paediatr Jpn. 1995; 37: 610613.

5. Shinde RB, Chauhan NM, Raut JS, Karuppayil SM. Sensitization of Candida albicans biofilms to various antifungal drugs by cyclosporine A. Ann Clin Microbiol Antimicrob. 2012; 11 (1): 27. doi: 10.1186/1476-0711-11-27.

6. Fortwendel JR, Juvvadi PR, Perfect BZ, Rogg LE, Perfect JR, Steinbach WJ. Transcriptional regulation of chitin synthases by calcineurin controls paradoxical growth of Aspergillus fumigatus in response to caspofungin. Antimicrob Agents Chemother. 2010; 54 (4): 1555-1563. doi: 10.1128/AAC.00854-09.

7. Stevens DA, White TC, Perlin DS, Selitrennikoff CP. Studies of the paradoxical effect of caspofungin at high drug concentrations. Diagn Microbiol Infect Dis. 2005; 51 (3): 173-178. doi: 10.1016/j.diagmicrobio.2004.10.006.

8. Cordeiro RA, Macedo RB, Teixeira CEC et al. The calcineurin inhibitor cyclosporin A exhibits synergism with antifungals against Candida parapsilosis species complex. J Med Microbiol. 2014; 63: 936-944. doi: 10.1099/ jmm.0.073478-0.

9. Lamoth F, Juvvadi PR, Gehrke C, Asfaw YG, Steinbach WJ. Transcriptional activation of heat shock protein 90 mediated via a proximal promoter region as trigger of caspofungin resistance in Aspergillus fumigatus. J Infect Dis. 2014; 209 (3): 473-481. doi: 10.1093/infdis/jit530.

10. Zhang C, Zhang J, Yang B, Wu C. Cyclosporin A inhibits the production of IL-17 by memory Th17 cells from healthy individuals and patients with rheumatoid arthritis. Cytokine. 2008; 42 (3): 345-352. doi: 10.1016/j.cyto.2008.03.006.

11. Rolain JM, Colson P, Raoult D. Recycling of chloroquine and its hydroxyl analogue to face bacterial, fungal and viral infections in the 21st century. Int J Antimicrob Agents. 2007; 30 (4): 297-308. doi: 10.1016/j.ijantimicag.2007.05.015.

12. Piconi S, Parisotto S, Rizzardini G et al. Hydroxychloroquine drastically reduces immune activation in HIV-infected, antiretroviral therapy-treated immunologic nonresponders. Blood. 2011; 118 (12): 3263-3272. doi: 10.1182/ blood-2011-01-329060.

13. Ornstein $\mathrm{MH}$, Sperber $\mathrm{K}$. The antiinflammatory and antiviral effects of hydroxychloroquine in two patients with acquired immunodeficiency syndrome and active inflammatory arthritis. Arthritis Rheum. 1996; 39 (1): 157-161. doi: 10.1002/art.1780390122.

14. Chang TH, Wang LF, Lin YS, Yang CS, Yu CY, Lin YL. Hydroxychloroquine activates host antiviral innate 
immunity. Cytokine. 2014; 70 (1): 33-34. doi: 10.1016/j. cyto.2014.07.032.

15. Byrd TF, Horwitz MA. Chloroquine inhibits the intracellular multiplication of legionella pneumophila by limiting the availability of iron: a potential new mechanism for the therapeutic effect of chloroquine against intracellular pathogens. J Clin Invest. 1991; 88 (1): 351-357. doi: 10.1172/JCl115301.

16. Mazzolla R, Barluzzi R, Brozzetti A et al. Enhanced resistance to Cryptococcus neoformans infection induced by chloroquine in a murine model of meningoencephalitis. Antimicrob Agents Chemother. 1997; 41 (4): 802-807.

17. Tsai WP, Nara PL, Kung HF, Oroszlan S. Inhibition of human immunodeficiency virus infectivity by chloroquine. AIDS Res Hum Retroviruses. 1990; 6 (4): 481-489. doi: 10.1089/aid.1990.6.481.

18. Chiang G, Sassaroli M, Louie M, Chen H, Stecher VJ, Sperber K. Inhibition of HIV-1 replication by hydroxychloroquine: mechanism of action and comparison with zidovudine. Clin Ther. 1996; 18 (6): 1080-1092. doi: 10.1016/S0149-2918(96)80063-4.

19. Paton NI, Goodall RL, Dunn DT et al. Effects of hydroxychloroquine on immune activation and disease progression among HIV-infected patients not receiving antiretroviral therapy. JAMA. 2012; 308 (4): 353-361. doi: 10.1001/jama.2012.6936.

20. Bishop NE. Examination of potential inhibitors of hepatitis A virus uncoating. Intervirology. 1998; 41 (6): 261-271. doi: 10.1159/000024948.

21. Keshavarzi F. Fungistatic effect of hydroxychloroquine, lessons from a case. Med Mycol Case Rep. 2016; 13: 17-18. doi: 10.1016/j.mmcr.2016.09.003.

22. Ciszek M, Mucha K, Foroncewicz B, Chmura A, Paczek L. Leflunomide as a rescue treatment in ganciclovir-resistant cytomegalovirus infection in a seronegative renal transplant recipient - a case report. Ann Transplant. 2014; 19 (1): 6063. doi: 10.12659/AOT.884035.

23. Lu CH, Tsai JH, Wu MZ, Yu CL, Hsieh SC. Can leflunomide play a role in cytomegalovirus disease prophylaxis besides its antirheumatic effects? Antivir Ther. 2015; 20 (1): 93-96. doi: 10.3851/IMP2796.

24. Avery RK, Mossad SB, Poggio E et al. Utility of leflunomide in the treatment of complex cytomegalovirus syndromes. Transplantation. 2010; 90 (4): 419-426. doi: 10.1097/ TP.0b013e3181e94106.

25. Waldman WJ, Knight DA, Blinder L, Shen J, Lurain NS, Miller DM. Inhibition of cytomegalovirus in vitro and in vivo. Intervirology. 1999; 42 (5-6): 412-428.

26. Cuellar-Rodriguez J, Stephany B, Poggio E et al. Contrasting patterns of viral load response in transplant recipients with BK polyomavirus DNAemia on leflunomide therapy. Clin Transplant. 2013; 27 (3). doi: 10.1111/ctr.12110.

27. Ritter ML, Pirofski L. Mycophenolate mofetil: Effects on cellular immune subsets, infectious complications, and antimicrobial activity: review article. Transpl Infect Dis. 2009; 11 (4): 290-297. doi: 10.1111/j.1399-3062.2009.00407.x

28. Fang S, Su J, Liang B et al. Suppression of autophagy by mycophenolic acid contributes to inhibition of HCV replication in human hepatoma cells. Sci Rep. 2017; 7: 1-12. doi: 10.1038/srep44039.

29. Ye L, Li J, Zhang T et al. Mycophenolate mofetil inhibits hepatitis $C$ virus replication in human hepatic cells. Virus Res. 2012; 168 (1-2): 33-40. doi: 10.1016/j.virusres.2012.06.009.
30. Pan Q, de Ruiter PE, Metselaar HJ et al. Mycophenolic acid augments interferon-stimulated gene expression and inhibits hepatitis $\mathrm{C}$ virus infection in vitro and in vivo. Hepatology. 2012; 55 (6): 1673-1683. doi: 10.1002/hep.25562.

31. Cho J, Yi H, Jang EY et al. Mycophenolic mofetil, an alternative antiviral and immunomodulator for the highly pathogenic avian influenza $\mathrm{H} 5 \mathrm{~N} 1$ virus infection. Biochem Biophys Res Commun. 2017; 494 (1-2): 298-304. doi: 10.1016/j.bbrc.2017.10.037.

32. Neyts J, Andrei G, De Clercq E. The novel immunosuppressive agent mycophenolate mofetil markedly potentiates the antiherpesvirus activities of acyclovir, ganciclovir, and penciclovir in vitro and in vivo. Antimicrob Agents Chemother. 1998; 42 (2): 216-222. doi: 10.1128/aac.44.6.1667-1673.2000.

33. Siebert A, Wysocka M, Krawczyk B, Cholewiński G, Rachoń J. Synthesis and antimicrobial activity of amino acid and peptide derivatives of mycophenolic acid. Eur J Med Chem. 2018; 143: 646-655. doi: 10.1016/j.ejmech.2017.11.094.

34. Dang W, Yin Y, Wang Y et al. Inhibition of calcineurin or IMP dehydrogenase exerts moderate to potent antiviral activity against norovirus replication. Antimicrob Agents Chemother. 2017; 61 (11). doi: 10.1128/AAC.01095-17.

35. Paravar T, Lee DJ. Thalidomide: Mechanisms of action. Int Rev Immunol. 2008; 27 (3): 111-135. doi: 10.1080/08830180801911339.

36. Chaulet $C$, Croix $C$, Alagille $D$ et al. Design, synthesis and biological evaluation of new thalidomide analogues as TNF- $\alpha$ and IL-6 production inhibitors. Bioorganic Med Chem Lett. 2011; 21 (3): 1019-1022. doi: 10.1016/j.bmcl.2010.12.031.

37. Hernandez MDO, Fulco TDO, Pinheiro RO et al. Thalidomide modulates Mycobacterium leprae-induced NF-kB pathway and lower cytokine response. Eur J Pharmacol. 2011; 670 (1): 272-279. doi: 10.1016/j.ejphar.2011.08.046.

38. Leite MR, Santos SS, Lyra AC, Mota J, Santana GO. Thalidomide induces mucosal healing in Crohn's disease: case report. World J Gastroenterol. 2011; 17 (45): 50285031. doi: 10.3748/wjg.v17.i45.5028.

39. Fu LM, Fu-Liu CS. Thalidomide and tuberculosis. Int $\mathrm{J}$ Tuberc Lung Dis. 2002; 6 (7): 569-572.

40. Tramontana JM, Utaipat U, Molloy $A$ et al. Thalidomide treatment reduces tumor necrosis factor alpha production and enhances weight gain in patients with pulmonary tuberculosis. Mol Med. 1995; 1 (4): 384-397.

41. Viel-Thériault I, Thibeault R, Boucher FD, Drolet JP. Thalidomide in refractory tuberculomas and pseudo abscesses. Pediatr Infect Dis J. 2016; 35 (11): 1262-1264. doi: $10.1097 /$ INF.0000000000001285.

42. Kawai T, Watanabe N, Yokoyama M et al. Thalidomide attenuates excessive inflammation without interrupting lipopolysaccharide-driven inflammatory cytokine production in chronic granulomatous disease. Clin Immunol. 2013; 147 (2): 122-128. doi: 10.1016/j.clim.2013.03.004.

43. Rosman Y, Lidiar M, Shoenfeld Y. Antibiotic therapy in autoimmune disorders. Clin Pract. 2014; 11 (1): 91-103. doi: $10.2217 / \mathrm{cpr} .13 .84$.

44. Lutz A. New ether oxime derivatives a structure-activity of erythromycin a relationship study. J Antibiot. 1990; 44 (3): 313-330.

45. Amsden GW. Anti-inflammatory effects of macrolides-An underappreciated benefit in the treatment of communityacquired respiratory tract infections and chronic inflammatory pulmonary conditions? J Antimicrob Chemother. 2005; 55 (1): 10-21. doi: 10.1093/jac/dkh519. 
46. Kipourou M, Manika K, Papavasileiou A et al. Immunomodulatory effect of macrolides: At what cost? Respir Med Case Reports. 2016; 17: 44-46. doi: 10.1016/j. rmcr.2016.01.004.

47. Ichiyama T, Nishikawa M, Yoshitomi T et al. Clarithromycin inhibits NF-kappaB activation in human peripheral blood mononuclear cells and pulmonary epithelial cells. Antimicrob Agents Chemother. 2001; 45 (1): 44-47. doi: 10.1128/ AAC.45.1.44.

48. Araujo FG, Slifer TL, Remington JS. Inhibition of secretion of interleukin-1 alpha and tumor necrosis factor alpha by the ketolide antibiotic telithromycin. Antimicrob Agents Chemother. 2002; 46 (10): 3327-3330. doi: 10.1128/ AAC.46.10.3327.

49. Ivetić-Tkalčević $V$, Bošnjak B, Hrvačić B et al. Antiinflammatory activity of azithromycin attenuates the effects of lipopolysaccharide administration in mice. Eur J Pharmacol. 2006; 539 (1-2): 131-138. doi: 10.1016/j. ejphar.2006.03.074.

50. Ratzinger F, Haslacher H, Poeppl W et al. Azithromycin suppresses CD4(+) T-cell activation by direct modulation of mTOR activity. Sci Rep. 2014; 4: 7438. doi: 10.1038/ srep07438.

51. Čulić O, Eraković V, Parnham MJ. Anti-inflammatory effects of macrolide antibiotics. Eur J Pharmacol. 2001; 429 (1-3): 209-229. doi: 10.1016/S0014-2999 (01)01321-8.

52. Thanou-Stavraki A, Aberle T, Aksentijevich I, Bane BL, Harley JB. Clarithromycin in adult-onset still's disease: a potentially useful therapeutic. J Clin Rheumatol. 2011; 17 (7): 373-376. doi: 10.1097/RHU.0b013e3182320680.

53. Saviola G, Abdi-Ali L, Rossini $P$ et al. Clarithromycin in rheumatoid arthritis patients not responsive to diseasemodifying antirheumatic drugs: an open, uncontrolled pilot study. Clin Exp Rheumatol. 2002; 20 (3): 373-378.

54. Saviola G, Benucci M, Abdi-Ali L et al. Clarithromycin in adult-onset Still's disease: a study of 6 cases. Rheumatol Int. 2010; 30 (4): 555-560. doi: 10.1007/s00296-009-1277-9.

55. Ogrendik M. Effects of clarithromycin in patients with active rheumatoid arthritis. Curr Med Res Opin. 2007; 23 (3): 515522. doi: 10.1185/030079906X167642.

56. Nanishi E, Nishio H, Takada $\mathrm{H}$ et al. Clarithromycin plus intravenous immunoglobulin therapy can reduce the relapse rate of Kawasaki disease: a phase 2, open-label, randomized control study. J Am Heart Assoc. 2017; 6 (7): 1-9. doi: 10.1161/JAHA.116.005370.

57. Täubel J, Lorch U, Rossignol JF, Ferber G, John Camm A. Analyzing the relationship of QT interval and exposure to nitazoxanide, a prospective candidate for influenza antiviral therapy-A formal TQT study. J Clin Pharmacol. 2014; 54 (9): 987-994. doi: 10.1002/jcph.300.

58. Rossignol JF. Nitazoxanide: a first-in-class broad-spectrum antiviral agent. Antiviral Res. 2014; 110: 94-103. doi: 10.1016/j.antiviral.2014.07.014.

59. Wang YM, Lu JW, Lin CC et al. Antiviral activities of niclosamide and nitazoxanide against chikungunya virus entry and transmission. Antiviral Res. 2016; 135: 81-90. doi: 10.1016/j.antiviral.2016.10.003.

60. Cao RY, Xu Y fen, Zhang TH et al. Pediatric drug nitazoxanide: a potential choice for control of Zika. Open Forum Infect Dis. 2017; 4 (1): 2-6. doi: 10.1093/OFID/ OFX009.

61. Nikolova K, Gluud C, Grevstad B, Jakobsen-Janus C. Nitazoxanide for chronic hepatitis C. Cochrane Database
Syst Rev. 2014; (4). doi: 10.1002/14651858.CD009182. pub2.

62. Kaufmann SHE, Dorhoi A, Hotchkiss RS, Bartenschlager R. Host-directed therapies for bacterial and viral infections. Nat Rev Drug Discov. 2018; 17 (1): 35-56. doi: 10.1038/ nrd.2017.162.

63. Di Santo N, Ehrisman J. A functional perspective of nitazoxanide as a potential anticancer drug. Mutat Res. 2014; 768: 16-21. doi: 10.1016/j.mrfmmm.2014.05.005.

64. Di Santo N, Ehrisman J. Research perspective: potential role of nitazoxanide in ovarian cancer treatment. old drug, new purpose? Cancers (Basel). 2013; 5 (3): 1163-1176. doi: 10.3390/cancers5031163.

65. Rozin A, Schapira D, Braun-Moscovici Y, Nahir AM. Cotrimoxazole treatment for rheumatoid arthritis. Semin Arthritis Rheum. 2001; 31 (2): 133-141. doi: 10.1053/sarh.2001.27734.

66. Stegeman CA, Tervaert JW, de Jong PE, Kallenberg CG. Trimethoprim-sulfamethoxazole (co-trimoxazole) for the prevention of relapses of Wegener's granulomatosis. Dutch Co-Trimoxazole Wegener Study Group. N Engl J Med. 1996; 335 (1): 16-20.

67. Tervaert JWC. Trimethoprim-sulfamethoxazole and antineutrophil cytoplasmic antibodies-associated vasculitis. Curr Opin Rheumatol. 2018; 30 (4): 388-394. doi: 10.1097/ BOR.0000000000000508.

68. Garrido-Mesa N, Zarzuelo A, Gálvez J. Minocycline: far beyond an antibiotic. Br J Pharmacol. 2013; 169: 337-352.

69. Szeto GL, Pomerantz JL, Graham DR, Clements JE. Minocycline suppresses activation of nuclear factor of activated T cells 1 (NFAT1) in human CD4+ T cells. J Biol Chem. 2011; 286 (13): 11275-11282.

70. Metz LM, Zhang Y, Yeung M, Patry DG, Bell RB, Stoian CA et al. Minocycline reduces gadolinium-enhancing magnetic resonance imaging lesions in multiple sclerosis. Ann Neurol. 2004; 55 (5): 756.

71. Metz LM, Li DKB, Traboulsee AL, Duquette P, Eliasziw M, Cerchiaro $\mathrm{G}$ et al. Trial of minocycline in a clinically isolated syndrome of multiple sclerosis. N Engl J Med. 2017; 376 (22): 2122-2133.

72. Gelfand EW. Intravenous immune globulin in autoimmune and inflammatory diseases. N Engl J Med. 2012; 367 (21): 2015-2025. doi: 10.1056/NEJMra1009433.

73. Berger M. Subcutaneous IgG in neurologic diseases. Immunotherapy. 2014; 6 (1): 71-83. doi: 10.2217/imt.13.146.

74. Bruton O. Agammaglobulinemia. Pediatrics. 1952; 9 (6): 722-728.

75. Imbach P, Barandun S, D'Apuzzo V et al. High-dose intravenous gammaglobulin for idiopathic thrombocytopenic purpura in childhood. Lancet. 1981; 317 (8232): 1228-1231. doi: 10.1016/S0140-6736 (81)92400-4.

76. Wahn V. From immune substitution to immunomodulation. Semin Hematol. 2016; 53: S7-S9. doi: 10.1053/j. seminhematol.2016.04.003.

77. Bozzo J, Jorquera JI. Use of human immunoglobulins as an anti-infective treatment: the experience so far and their possible re-emerging role. Expert Rev Anti Infect Ther. 2017; 15 (6): 585-604. doi: 10.1080/14787210.2017.1328278.

78. Rena G, Pearson ER, Sakamoto K. Molecular mechanism of action of metformin: old or new insights? Diabetologia. 2013; 56 (9): 1898-1906. doi: 10.1007/s00125-013-2991-0.

79. Pollak M. The effects of metformin on gut microbiota and the immune system as research frontiers. Diabetologia. 2017; 60 (9): 1662-1667. doi: 10.1007/s00125-017-4352-x. 
80. Lee SY, Moon SJ, Kim EK et al. Metformin suppresses systemic autoimmunity in roquin san/san mice through inhibiting $B$ cell differentiation into plasma cells via regulation of AMPK/mTOR/STAT3. J Immunol. 2017; 1403088. doi: 10.4049/jimmunol.1403088.

81. Restrepo BI. Metformin: candidate host-directed therapy for tuberculosis in diabetes and non-diabetes patients. Tuberculosis. 2016; 101: S69-S72. doi: 10.1016/j. tube.2016.09.008.

82. Singhal A, Jie L, Kumar $P$ et al. Metformin as adjunct antituberculosis therapy. Sci Transl Med. 2014; 6 (263). doi: 10.1126/scitranslmed.3009885.

83. Lee YJ, Han SK, Park JH et al. The effect of metformin on culture conversion in tuberculosis patients with diabetes mellitus. Korean J Intern Med. 2018; 33 (5): 933-940.

84. Marupuru S, Senapati P, Pathadka S, Miraj SS, Unnikrishnan MK, Manu MK. Protective effect of metformin against tuberculosis infections in diabetic patients: an observational study of south Indian tertiary healthcare facility. Brazilian J Infect Dis. 2017; 21 (3): 312-316. doi: 10.1016/j.bjid.2017.01.001.

85. Abeles AM, Pillinger MH. Statins as antiinflammatory and immunomodulatory agents: a future in rheumatologic therapy? Arthritis Rheum. 2006; 54 (2): 393-407. doi: 10.1002/art.21521.

86. Lobato LS, Rosa PS, Da Silva Ferreira J et al. Statins increase rifampin mycobactericidal effect. Antimicrob Agents Chemother. 2014; 58 (10): 5766-5774. doi: 10.1128/ AAC.01826-13.

87. Mehrbod P, Omar AR, Hair-Bejo M, Haghani A, Ideris A. Mechanisms of action and efficacy of statins against influenza. Biomed Res Int. 2014; 2014: 11-14. doi: 10.1155/2014/872370.
88. Yamazaki H, Suzuki M, Aoki T et al. Influence of 3-hydroxy3-methylglutaryl coenzyme a reductase inhibitors on ubiquinone levels in rat skeletal muscle and heart: relationship to cytotoxicity and inhibitory activity for cholesterol synthesis in human skeletal muscle cells. $J$ Atheroscler Thromb. 2006; 13 (6): 295-307. doi: 10.5551/ jat.13.295.

89. Masadeh M, Mhaidat N, Alzoubi K, Al-Azzam S, Alnasser Z. Antibacterial activity of statins: a comparative study of atorvastatin, simvastatin, and rosuvastatin. Ann Clin Microbiol Antimicrob. 2012; 11: 1-5. doi: 10.1186/14760711-11-13.

90. Tapia-Pérez JH, Kirches E, Mawrin C, Firsching R, Schneider T. Cytotoxic effect of different statins and thiazolidinediones on malignant glioma cells. Cancer Chemother Pharmacol. 2011; 67 (5): 1193-1201. doi: 10.1007/s00280-010-1535-2.

91. Schramm R, Menger MD, Harder $Y$ et al. Statins inhibit lymphocyte homing to peripheral lymph nodes. Immunology. 2007; 120 (3): 315-324. doi: 10.1111/j.13652567.2006.02505.x

92. Fernandez-Boyanapalli RF, Frasch SC, Thomas SM et al Pioglitazone restores phagocyte mitochondrial oxidants and bactericidal capacity in chronic granulomatous disease. $J$ Allergy Clin Immunol. 2015; 135 (2): 517-527. doi: 10.1016/j. jaci.2014.10.034.

\section{Correspondencia:}

Dr. Marco Antonio Yamazaki-Nakashimada

Insurgentes Sur 3700, Letra C,

Col. Insurgentes Cuicuilco,

Alcaldía Coyoacán, 04530 ,

Ciudad de México, México.

E-mail: yzki71@yahoo.com.mx 